\title{
The National Ignition Facility: the world's largest optical system
}

\section{Christopher Stolz}

Christopher J. Stolz, "The National Ignition Facility: the world's largest optical system," Proc. SPIE 6834, Optical Design and Testing III, 683402 (28 November 2007); doi: 10.1117/12.773365

SPIE. Event: Photonics Asia 2007, 2007, Beijing, China 


\title{
The National Ignition Facility: The world's largest optical system
}

\author{
Christopher J. Stolz \\ University of California, Lawrence Livermore National Laboratory, \\ 7000 East Avenue L-491, Livermore, CA 94550
}

\begin{abstract}
The National Ignition Facility (NIF), a 192-beam fusion laser, is presently under construction at the Lawrence Livermore National Laboratory with an expected completion in $2008 .^{1-5}$ The facility contains 7,456 meter-scale optics for amplification, beam steering, vacuum barriers, focusing, polarization rotation, and wavelength conversion. ${ }^{6} \mathrm{~A}$ multiphase program was put in place to increase the monthly optical manufacturing rate by up to $20 \times$ while simultaneously reducing cost by up to $3 \times$ through a sub-scale development, full-scale facilitization, and a pilot production phase. Currently $80 \%$ of the optics are complete with over $50 \%$ installed. In order to manufacture the high quality optics at desired manufacturing rate of over 100 precision optics per month, new more deterministic advanced fabrication technologies had to be employed over those used to manufacture previous fusion lasers.
\end{abstract}

Keywords: Laser glass, fused silica, KDP, DKDP, laser resistance, mirrors, polarizers, optical thin films

\section{INTRODUCTION}

In 2010, fifty years after the invention of the laser, the NIF will achieve fusion ignition. The NIF is the latest in a series of increasingly more powerful lasers in the quest to achieve for the first time in a controlled laboratory setting, the conditions necessary for ignition. ${ }^{x}$ The path to laser fusion has relied heavily on significant advances in optical technologies to facilitate lasers with ever increasing energies. Some of the more significant changes between NIF and Nova, the preceding fusion laser at LLNL, are rapid growth for KDP and large-aperture conventional growth of DKDP crystals, continuous pour technology of a reformulated laser glass, deterministic grinding and finishing technologies, deterministic coating deposition processes, deformable mirrors, and finally laser processing of all high fluence optics. ${ }^{6}$

\section{LASER GLASS}

NIF laser glass illustrated in figure 1 is a neodymium doped phosphate glass that is pumped with flash lamps to achieve laser gain. In the NIF laser 15 orders of magnitude of gain is achieved through the use of this glass. Prior to NIF all the laser glass poured for previous fusion lasers was done by a discontinuous pour process where each glass blank is poured into a mold. Run-to-run variations in processing conditions impacted quality and this fabrication technique yields only a few slabs per week. Because of the large number of slabs (3072) required for NIF, continuous pour technologies utilized for other commercial glasses were developed specifically for laser glass. New melters were manufactured to meet the unique characteristics of laser glass. ${ }^{7}$ These melters could achieve melt rates of 70-300 slabs per week. In fact our slab blank manufacturing rate is limited by the post processing shaping and prefinishing of the glass needed to verify the homogeneity and laser resistance.

Homogeneity is measured with custom 24 inch $(61 \mathrm{~cm})$ aperture phase measuring interferometers that were fielded at each of the NIF optical fabrication vendors to validate wavefront performance. Laser resistance is determined by scanning the optic past a $1064 \mathrm{~nm} 10 \mathrm{~ns}$ pulse length Gaussian laser beam. During this process residual platinum particles from platinum that lines the melter, that are imbedded in the laser glass are initiated. In order to utilize as many slabs as possible, slabs with platinum sites are used in low fluence locations within the amplifier where the sites will remain stable. In the high fluence locations, only platinum free laser glass is used due to it's high laser resistance.

After the glass is preformed and metrology is completed, the slabs are shaped to their final dimensions, prepolished, and final figured as described in section 4.1 below. As part of the finishing process, copper doped phosphate glass cladding strips are epoxied to the slab perimeter. This cladding is to absorb stray light within the slab so that it does not amplify thus creating damage to slab edges and reducing the amplification in the direction of the NIF beam.

Optical Design and Testing III, edited by Yongtian Wang, Theo T. Tschudi, Jannick P. Rolland, Kimio Tatsuno Proc. of SPIE Vol. 6834, 683402, (2007) · 0277-786X/07/\$18 · doi: 10.1117/12.773365 

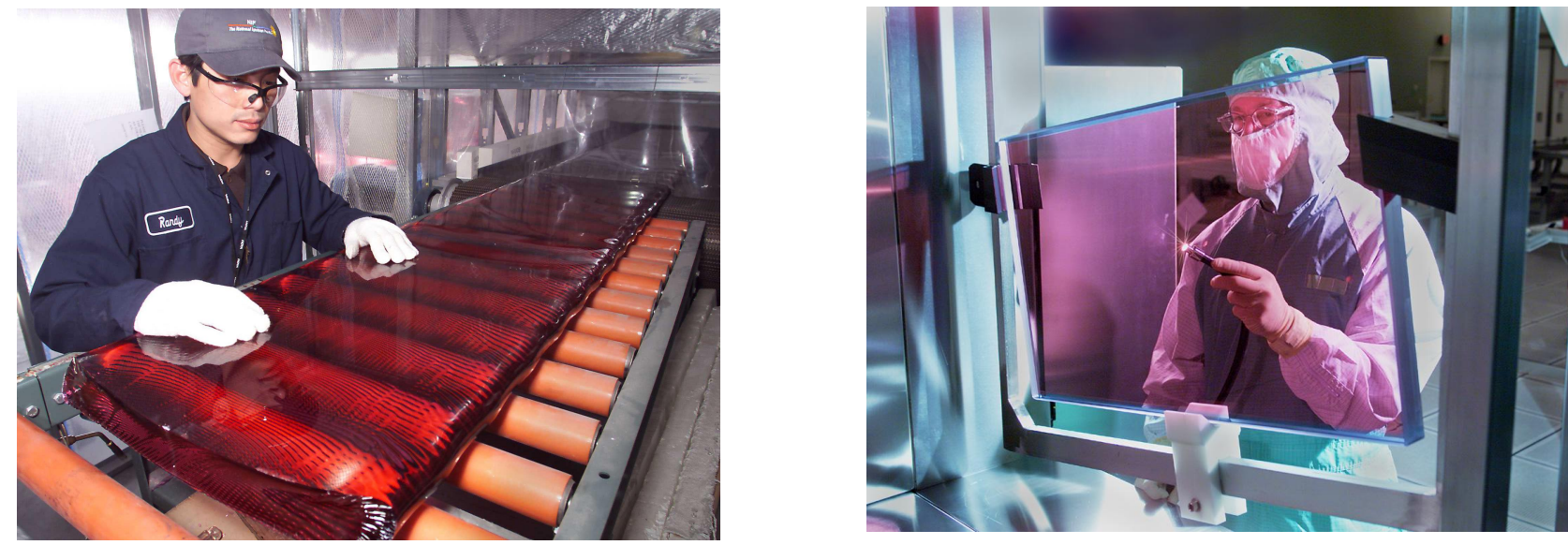

Fig. 1 Continuous strip of laser glass exiting the melter (left image) and completed laser glass slab undergoing inspection after cleaning (right image).

\section{KDP CRYSTALS}

Potassium dihydrogen phosphate (KDP) pockels cells have been used on multiple fusion laser systems for isolation. Size limitations prevented their use on Nova before final beam expansion. The NIF laser uses a full-aperture Pockel's cell for both isolation and pulse switching in the main laser cavity. The advantage of this multi-pass approach is the utilization of optics in 4 passes through the amplifier thus significantly reducing the number of amplifier slabs, flash lamps, spatial filter lenses. Additionally the overall facility has a smaller foot print due to the multi-pass architecture. In order to achieve uniform beam rotation over a $40 \mathrm{~cm} \times 40 \mathrm{~cm}$ beam, a plasma-electrode pockels cell (PEPC), was designed. ${ }^{\mathrm{x}}$ Today 192 PEPCs are installed on NIF and are operational.

The Nova laser was the first fusion laser to be frequency converted. Because available crystals were smaller than the Nova beam, an array of crystals were used in each beamline to triple the frequency of the laser. To achieve higher conversion efficiency, single crystals are used on the NIF laser. In fact the size of crystals was one of the design constraints on the beam dimensions of NIF. Unfortunately it takes up to 3 years to grow crystals of adequate size to harvest multiple plates with dimensions of $40 \mathrm{~cm} \times 40 \mathrm{~cm}$. A rapid growth development program was started at LLNL. ${ }^{\mathrm{x}}$ Today KDP crystals as shown in figure 2 are grown to sizes exceeding 380 kilograms in only 3 months. The rapid growth process is achieved by controlling the growth rate in all three axis in a super saturated solution to yield the desired crystal geometry for maximum yield of the crystalline plates. In conventional growth, the crystal only grows in the $\mathrm{Z}$-axis requiring large starting seeds and much lower overall crystal growth rates.

Once the crystals are grown, they are cut into large plates and single-point diamond turned to achieve the final figure and transmitted wavefront performance. Diamond turning machines were designed and fabricated to figure full size crystals. ${ }^{\mathrm{x}}$ Metrology tools were also put into place to measure the wavefront, crystal orientation, and retardation (for full aperture waveplates).

\section{DETERMINISTIC FINISHING TECHNOLOGIES}

Conventional optical fabrication technologies rely heavily of manual fabrication processes and iterative figure control. Typically an optic is polished on a lap, measured, analyzed to determine how close the optic is to specification, and put back on a lap. This process is repeated until the optic finally meets specification. The skill of the optician is critical in reducing the number of iterations, however, the process is not very deterministic. It was these traditional manufacturing techniques that were employed on all of the previous fusion lasers. Because the number of NIF optics is so large, deterministic processes were developed. 


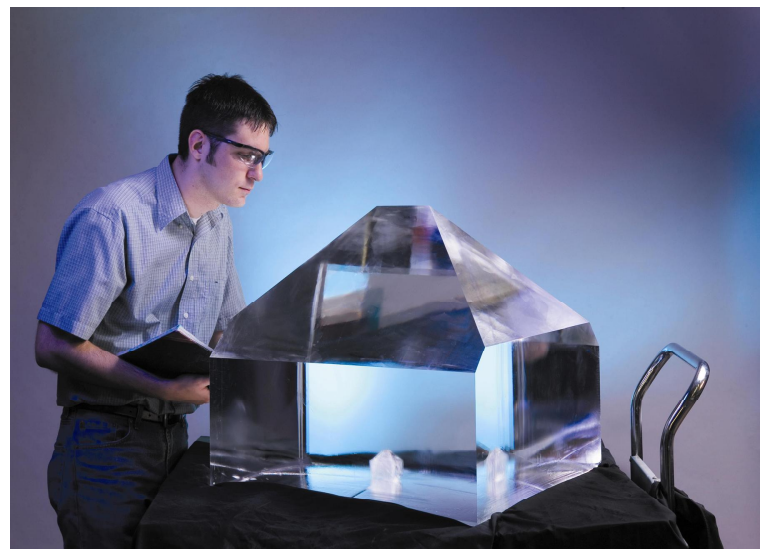

Fig 2. KDP doubler crystal grown using the rapid growth technique (left image). Final assembly of the PEPC utilizing KDP to rotate the beam polarization as part of an optical switch (right image).

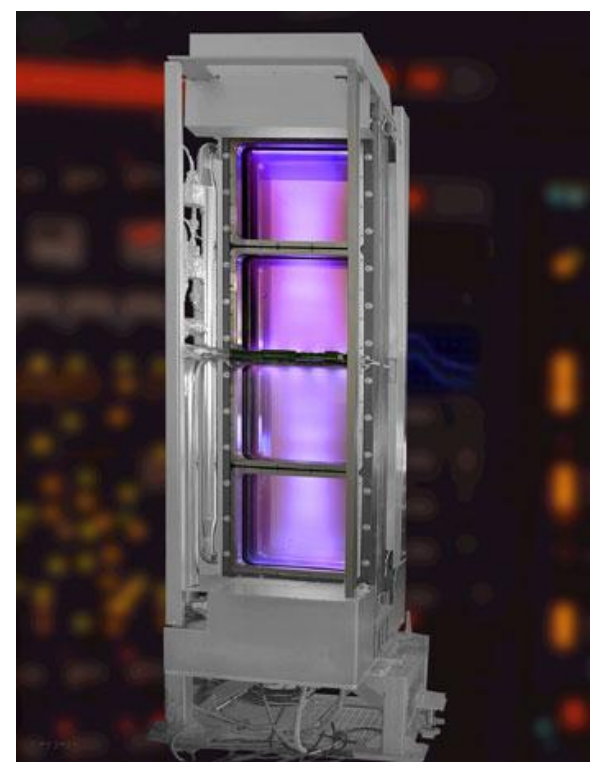

\subsection{FLATS FINISHING}

Improvements were made to both the grinding and polishing area in order to realize a more deterministic process. In the griding area, prior to NIF all of the large optics were loose abraisively ground, a manually intensive process. To reduce grinding time fixed abraisive grinding techniques were investigated. Specifically, electrolytic in-situ dressing (ELID) was scaled to NIF apertures and is now routinely performed on amplifier slabs and BK7 mirrors and polarizers. The ELID process is a method to continuously expose fresh diamonds on a grinding wheel to maximize the grinding rate and to reproducably generate a very flat surface thus minimizing the amount of high speed polishing. The grinding wheel is exposed to a current which accelerates the rusting process. This accomplishes two things, it minimizes the buildup of small glass particles (much like the build up of sawdust on sandpaper) thus elliminating the need for constantly redressing the wheel that leads to difficulty in figure control. The rusting also exposes new diamonds so that the the wheel does not dull.

NIF optics are prepolished with high speed synthetic polishing machines to remove grinding-induced damage, correct wedge, and achieve moderate flatness to minimize the amount of final figuring needed to achieve microroughness and final flatness specifications. Ring polishers (also referred to as continuous polishers) of over 4 meters in diameter were constructed to polish all of the amplifier slabs, mirrors, and polarizers. Unique computer controls were integrated into these polishing machines to enhance control of the lap surface flatness to minimize the number of testing iterations before optic acceptance.

\subsection{COMPUTER CONTROLLED FINISHING}

Computer-controlled small-tool figuring technologies had been used extensively before NIF to fabricate aspheric lenses. However, for NIF the processes were optimized to yield high laser resistant optical surfaces exposed to $351 \mathrm{~nm}$ laser light. A very small portion of the laser glass slabs have material inhomogeneity that prevents the use of traditional lap polishing to meet the transmitted wavefront specification. Small-tool computer controlled finishing has been a useful technique to generate an aspheric surface to overcome the transmitted wavefront distortion caused by the material inhomogeneity. Small-tool figuring can create microripples on the surface. PSD specifications were determined to prevent downstream light intensification from surface microripples. This led to a development program with small-tool figuring vendors to develop a process that minimizes surface ripples to yield low PSD surfaces.

Ion-figuring is an additional small-tool figuring technique that has been utilized for NIF optics to overcome material inhomogeneity impacts on transmitted wavefront. The BK7 polarizers on NIF are quite thick $(9 \mathrm{~cm})$ and large $(40 \mathrm{~cm} \times$ $80 \mathrm{~cm}$ ). To achieve a peak-to-valley transmitted wavefront of $\lambda / 3$ (waves) and RMS gradient of $\lambda / 90$ (waves/cm), a 
material inhomogeneity specification of better than $+/-1 \mathrm{ppm}$ is required. Material availability and cost were both prohibative to obtain material of this purity over the NIF polarizer dimensions for 200 optics. Since the polarizers are used in both reflection and transmission, the exit surface can be aspherized with ion figuring to realize a transmitted wavefront that is typically twice as good as the specification for a cost that is lower than the differential cost associated with purchasing higher homogeneity BK7.

\subsection{MAGNETORHEOLOGICAL FINISHING}

In order to change the spot size of the beam on target, a full-aperture phase plate will be inserted in front of each of the 192 NIF beams. For each experiment where a different beam dimension is needed on target, a new set of phase plates will be constructed. The Laboratory for Laser Energetics has developed an in-house ion etching process to manufacture the surface profile that breaks up the coherence of the beam to create a uniform flat top beam. LLNL has selected magnetorheological finishing (MRF) to manufacture phase plates. The NIF close-packed architecture within the final optics assembly dictates that surface flaws that can occur with ion-etching are unacceptable.

MRF is a highly deterministic small-tool polishing technique that has recently been scaled to 1 meter aperture optics. An iron based polishing slurry is passed over a magnetic field creating a stable small tool profile with a constant stream of fresh slurry. A CNC base is used for precise control. The material removal is determined by dwell time over the polishing head. Interferometry data is measured and compared to the surface prescription of the phase plate surface profile design. A difference profile (or hit map) is generated for rapid convergence to the desired surface profile.

An additional advantage of MRF is that the polishing is done in shear as opposed to compression for conventional polishing techniques. This difference in polishing methodology minimizes polishing-induced subsurface damage. Therefore, MRF has been utilized to polish wedges into optical surfaces to characterize the depth of grinding-induced subsurface damage. ${ }^{x}$ This characterization has been critical to determine removal rates during different grinding operations to yield subsurface damage free optics necessary for high laser resistance at $351 \mathrm{~nm}$. Finally MRF polishing can also be used to fabricate high UV laser resistance when the surface is acid etched to remove residual iron from the polishing slurry. ${ }^{\mathrm{x}}$
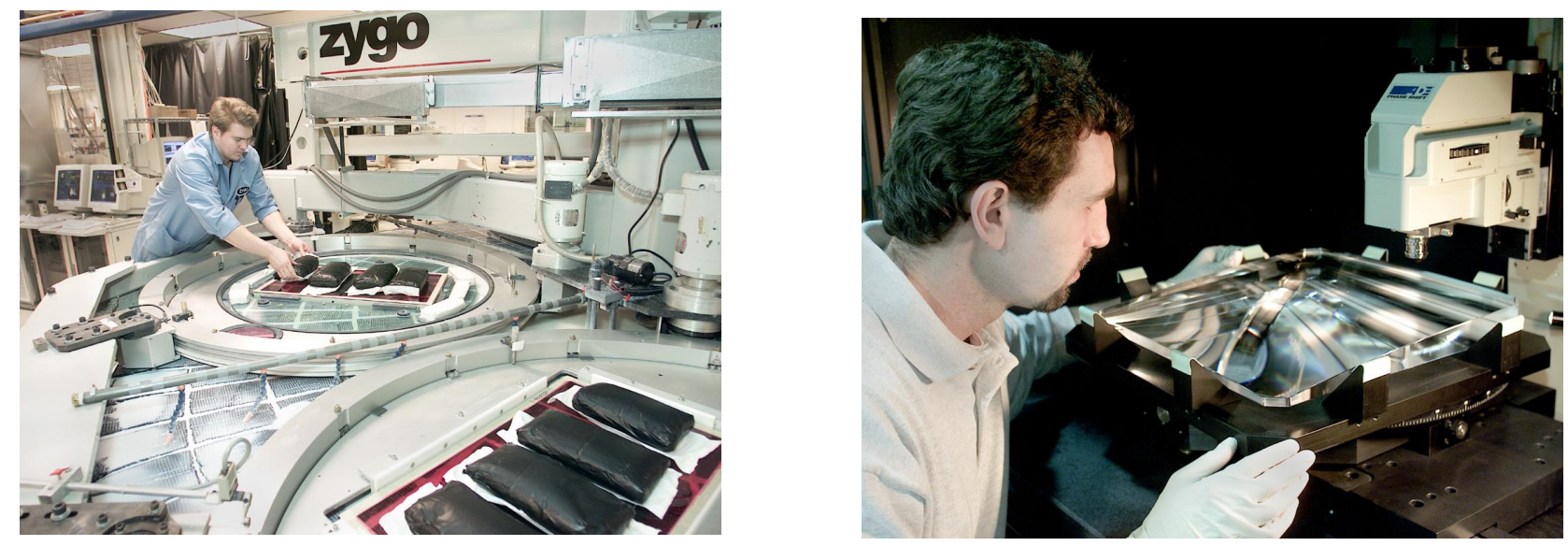

Fig. 3 One of three 168 inch diameter ring polishing used to deterministically final figure NIF laser glass and BK7 optics (left image). Wedged off-axis aspheric final focus lens under inspection for PSD and microroughness (left image).

\section{OPTICAL COATINGS}

The focus of the NIF coating development was to improve the deposition technique so that it would become more deterministic to improve spectral and wavefront yield as well as defect minimization to increase laser resistance. Therefore, the major emphasis was on source stabilization and improved in-situ metrology. Source stabilization was primarily accomplished by using $\mathrm{Hf}$ metal instead of $\mathrm{HfO}_{2} .{ }^{\mathrm{x}}$ The higher thermal conductivity of the metallic source 
allowed the use of a stationary e-beam and a large molten surface without the traditional tunneling or drilling problems seen with oxide starting materials. A side benefit of the source material switch was improved laser resistance through reduced defect densities and improved interfacial quality. The use of multiple crystal rate monitors within the deposition chamber was also used to determine the deposition plume stability instead of just monitoring deposition rate at a single location. With these additional monitors, sweep parameters could be determined that resulted in the most stable deposition plume.

Optical coatings also play a pivotal role in the stability of the high-gain $\left(10^{9}\right)$, small-aperture $(<50 \mathrm{~mm})$ amplifiers on NIF. Polarizers are used to trap and extract the pulse within the amplifiers. The polarizers and high fluence mirrors were manufactured by ion beam sputtering (IBS), a dense thin film process that is spectrally-stable with environmental changes. E-beam coatings were used solely for mirrors and polarizers on all of the previous LLNL fusion lasers.

\subsection{SOLARIZATION FOR STRAY LIGHT ARMORING}

The backscattered light from the fusion target will strike the $1053 \mathrm{~nm}$ transport mirrors. The spectral wavelengths of the backscattered light cover $351 \mathrm{~nm}$ (Stimulated Brillion Scattering) and 400 - $700 \mathrm{~nm}$ (Stimulated Raman Scattering). To prevent upstream damage within the NIF laser, the transport mirror coatings have been designed to transmit the SBS and SRS wavelengths through the coatings while being a high reflector at $1053 \mathrm{~nm}$ for beam transport and $374 \mathrm{~nm}$ for optic alignment through the final optics. Unfortunately, the roughly $1 \mathrm{~J} / \mathrm{cm}^{2}$ of backscattered energy is high enough to cause surface degradation to the metal structure that supports the transport mirrors. The solution to this problem was to change the absorption of the BK7 glass mirror substrate via solarization or color center formation by exposure to gamma radiation from a Cobalt source. ${ }^{\mathrm{X}}$ The spectra of the solarization covers the full visible range, although the absorptance is less as the wavelength increases.

Since the e-beam coatings are deposited at elevated temperature which would lead to solarization bleaching (color center annihilation), the BK7 solarization has to occur after coating. Testing validated that the solarization had no negative impact on the laser resistance of laser conditioned coatings. Accelerated bleaching rate tests were conducted at a variety of temperatures to develop a model to estimate the bleaching rate at ambient temperature. The model suggests annihilation of the color centers is a quite slow at the NIF facility operating temperature $(20 \mathrm{deg}$. C) resulting in at least 10 years of operation before additional solarization would be needed. This is consistent with the expected lifetime of NIF transport mirrors.

\subsection{GAS KNIVES FOR CONTAMINATION CONTROL}

Contamination control is critical for high fluence laser systems. Laser studies of contaminated mirrors exposed to a variety of particles compositions (metals, silicates, and organics) over a range of sizes (tens to hundreds of microns in diameter) showed a significant reduction in coated mirror operational lifetime ${ }^{-\mathrm{x}}$ In order maximize NIF mirror lifetimes, small particles with diameters equal to or larger than 50 microns must be removed before installation into the NIF laser. Unfortunately particles can contaminate a pristine optical surface from either airborne debris or localized shedding of particles from surrounding mounting hardware, actuator motors, welding seams in beam tubes, etc. One technique to remove surface particles in-situ is with a gas knife.

Air knives consist of a tube that extends along the length of an optic. A narrow slit along the tube is used to blow a high velocity laminar burst of air over the optical surface at a glancing incidence angle with the intent of dislodging surface particles over the entire surface. After extensive testing of a prototype device in a beam enclosure, it was validated that with multiple bursts over $90 \%$ of the particles are permanently removed. One question that needed answering was "Where do the particles go after being dislodged from the mirror surface?". To address this concern, polystyrene spheres were sprinkled over a mirror surface and examined under black light due to their fluorescence to see where they land after being exposed to a burst of gas. Some particles simply fall back on the mirror surface, hence the requirement for the multiple gas knife bursts. The majority of particles succumb to gravity and fall to the base of the beam enclosure. 


\subsection{FULL-APERTURE DEFORMABLE MIRRORS}

The NIF beams are focused into a 600 micron spot. To achieve this requirement requires control of two beam parameters, pointing stability and beam diameter. Deformable mirrors are used to predominately control the latter requirement. In the NIF laser, there are 3 main sources of wavefront deformation. Each of the optics in the laser beam have imperfect flatness or departure from the prescribed aspheric surface in the case of the lenses and minor inhomogeneity in the transmissive optical components such as windows, laser glass slabs, and polarizers. In the case of thick $(90 \mathrm{~mm})$ polarizers, a good portion of the transmitted wavefront error due to material inhomogeneity is corrected by ion figuring. The second wavefront error that impacts beam focusability is pump-induced distortion. This effect is caused by laser glass slab heating when the flashlamps are on which causes this effect. The final dominant wavefront distortion term is due to residual heat in the slabs after the flashlamps have been fired. A desire to maximize the shot rate on NIF (up to 1 shot per hour) drives this requirement. Air cooling has also been added to the NIF amplifier section in order to minimize thermal distortion.

Deformable mirror technology was first demonstrated on Beamlet, as single-beam prototype of the NIF architecture. Because the Beamlet deformable mirror borrowed heavily from AVLIS technology, it was a sub-aperture mirror that was placed after one of the small-aperture amplifiers, just before the beam expansion. A full-aperture $(40 \mathrm{~cm} \times 40 \mathrm{~cm})$ prototype was fielded on Beamlet to demonstrate performance before launching the production design. Today nearly 200 deformable mirrors have been manufactured and installed on NIF.

The production mirror has thirty-nine actuator posts and two alignment ports that are $\mathrm{CNC}$ machined out of a monolithic piece of BK7. A metal reaction block supports the actuators rigidly to 39 separate machined flexures. The reaction block and glass mirrors are epoxied together under an interferometer to monitor the DFM wavefront during bonding and curing to validate compliance with specification after curing. Finally mirrors are characterized after bonding as illustrated in figure 4.
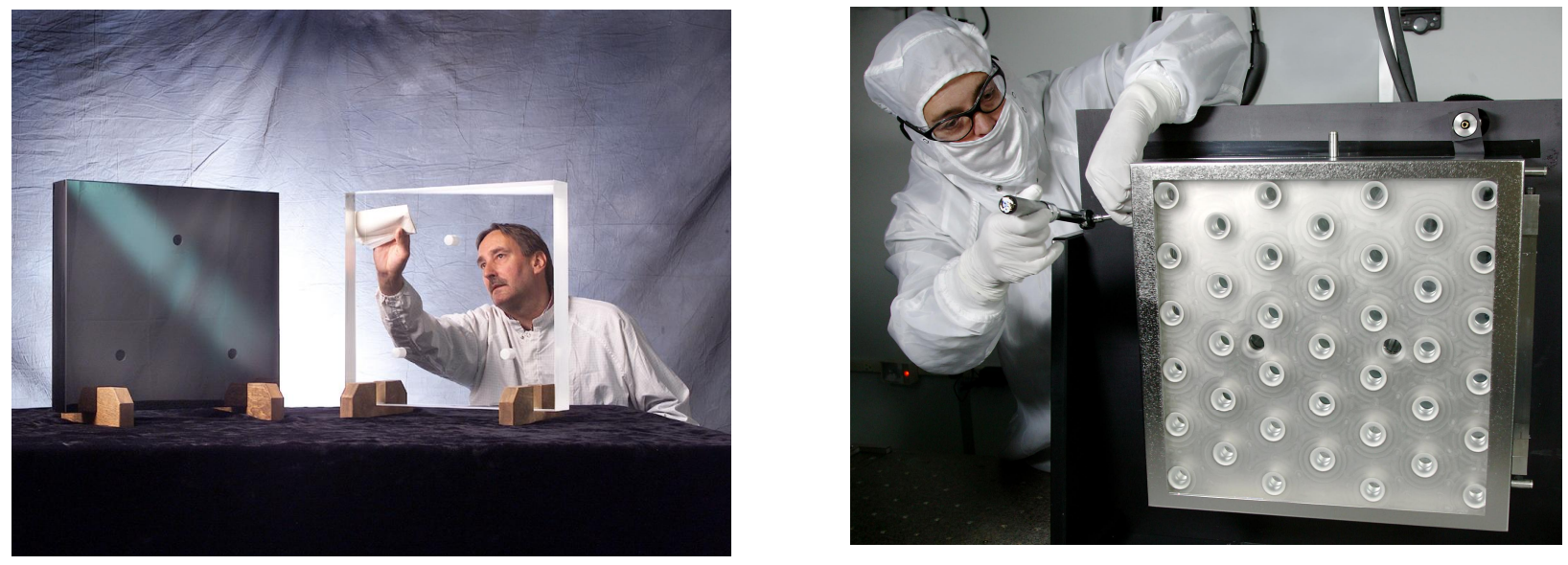

Fig. 4 Example of an unsolarized and solarized transport mirror (left image). NIF deformable mirror undergoing wavefront validation testing (right image).

\section{IMPROVING LASER RESISTANCE AND OPTIC LIFETIME}

The optics in the NIF laser are exposed to fluences that are $5-8 \times$ higher than in Nova. To meet this performance requirement, high fluence optics are laser processed. The only optics for Nova that were laser processed were the laser glass slabs to expose the platinum particles as described in section 2. One of the laser based post processing steps is laser conditioning, a process were an optic is exposed to progressively higher fluence in a gentle ramp. Laser conditioning typically increases the threshold of an optic by at least $2 \times$.

The KDP and DKDP crystals are laser conditioned to increase the laser resistance of the bulk material. It was found that KDP crystals that operate at $1053 \mathrm{~nm}$ realize higher laser resistance when laser conditioned in the UV. Conditioning stations were built and are currently being operated at LLNL to excimer laser condition the large aperture pockel cell 
crystals (switch crystals). KDP doublers are conditioned on NIF since each beam is being slowly ramped up in fluence as part of the commissioning process. For DKDP triplers it was found that the optimum laser conditioning occurs at 351 $\mathrm{nm}$ with a subnanosecond pulse length.

Multilayer coatings (mirrors and polarizers) are also laser conditioned with stations that were fielded at the NIF vendors as illustrated in figure 5 . The high fluence $1053 \mathrm{~nm}$ transport mirrors are scan at 3 progressively higher fluences in order to gently dislodge absorbing defects, leaving benign nodule ejection pits. In addition to increasing the laser resistance, the laser conditioning stations are also a full-aperture laser damage threshold validation metrology tool so that only the highest fluence optical coatings are installed on NIF. Finally, the laser conditioning stations have plasma and scatter detectors to determine the amount of surface modification. Recent studies have shown that plasma scalds created during laser exposure can cause micro-roughening of the surface resulting in beam contrast enhancements on downstream optics. $^{x}$

The second post-processing technique is called mitigation. This is a surface technique that is used on a laser-induced fracture site, where the site is modified to increase its laser resistance. In some ways this is like a surface microsurgery technique. This technique is used for fused silica and KDP surfaces. The success of this technique is due to realization of low subsurface damage optics, thus only a few initiation site occur at the NIF operational fluences. Of equal importance is the development of surface treatments that removed the laser-induced surface cracks by either a high speed diamond drill for crystals or $\mathrm{CO}_{2}$ melting processes that melt away the cracked area for silica optics (figure 5). To mitigate a surface, the optic is prescanned, much like laser conditioning. Low density surface defects are initiated. $\mathrm{CO}_{2}$ lasers or diamond drills are then used to remove the initiated damage. Because the NIF laser has a built in damage inspection system, optics that are damage during operation will be removed while the initiation sites are still small so that they can be mitigated. This will minimize the number of refinishing cycles and replacement optics during NIF operations.
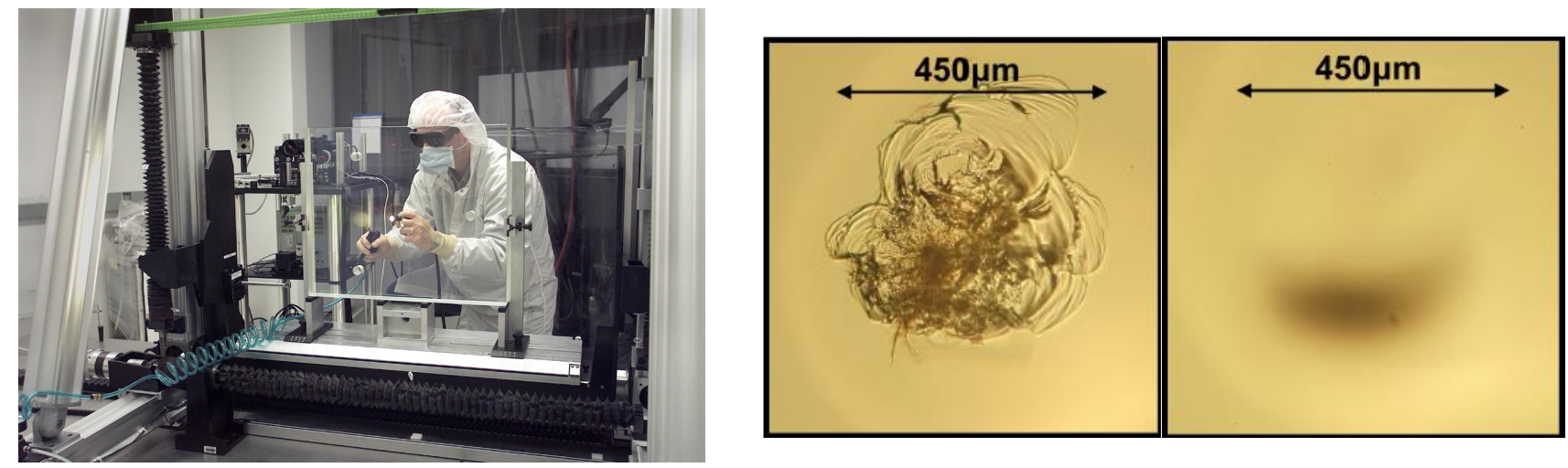

Fig. 5 NIF multilayer transport mirror being inspected after laser conditioning (left image). Crack elimination in a fused silica initiation site which has been mitigated by a doubled $\mathrm{CO}_{2}$ laser (right image).

\section{FUTURE TRENDS}

The push for future fusion laser systems will no longer be on ignition demonstration, but toward the technology development of facilities with high repetition rates $(>360 \times)$ and high electrical efficiency $(10-20 \times)$ needed for commercialization of fusion power plants. In the area of optics, continued advances are expected to occur in the areas of material developments to yield higher purity materials to construct optics and coatings to increase laser resistance and optic lifetime. Similarly, finishing technologies are also expected to continue to advance to minimize surface defects so that fewer initiation sites are created that require mitigation. Advances in crystalline materials for amplification over large apertures would increase the electrical to laser light conversion efficiency. Currently materials such as ceramic crystals or high temperature growth S:FAP are particularly interesting. Obviously optical fabrication capacity will need to increase drastically to meet the needs of commercial fusion plant builds. 
An additional development area is with short pulse (picoseconds) Petwatt lasers that can be used to reduce the necessary target compression. By pulse stretching and compression techniques, long pulse amplifiers can be used to created the needed energy while high fluence gratings within the final optics assembly can be used to achieve the pulse compression. The production of high fluence multi-layer gratings remains a very active area of research.

\section{CONCLUSIONS}

The optics in the National Ignition Facility have been possible because of technology developments that have occurred within the optics industry for both the advancement of fusion lasers as well as other commercial ventures. These advances include continuous pour laser glass, rapid growth KDP and large-aperture DKDP growth, and improvements in material, finishing, and coating processes through increased determinism. Finally laser processing of all high fluence optics with mitigation or microsurgery of initiation sites has significantly raised laser resistance and operational lifetime of optical components.

\section{AKNOWLEDGEMENTS}

The author would like to acknowledge the support of Jenessa Huffman in assisting with this manuscript. This article summarizes the work of countless individuals and work teams dedicated to the technology development and construction of these truly amazing laser facilities. This work is performed under the auspices of the U.S. Department of Energy by Lawrence Livermore National Laboratory under Contract DE-AC52-07NA27344.

\section{DISCLAIMER}

This document was prepared as an account of work sponsored by an agency of the United States government. Neither the United States government nor Lawrence Livermore National Security, LLC, nor any of their employees makes any warranty, expressed or implied, or assumes any legal liability or responsibility for the accuracy, completeness, or usefulness of any information, apparatus, product, or process disclosed, or represents that its use would not infringe privately owned rights. Reference herein to any specific commercial product, process, or service by trade name, trademark, manufacturer, or otherwise does not necessarily constitute or imply its endorsement, recommendation, or favoring by the United States government or Lawrence Livermore National Security, LLC. The views and opinions of authors expressed herein do not necessarily state or reflect those of the United States government or Lawrence Livermore National Security, LLC, and shall not be used for advertising or product endorsement purposes.

\section{REFERENCES}

1. E. Moses, et al., Fusion Science and Technology, 43, p. 420, May 2003.

2. E. I. Moses, J. H. Campbell, C. J. Stolz, and C. R. Wuest, "The National Ignition Facility: the world's largest optics and laser system," in Optical Engineering at the Lawrence Livermore National Laboratory, T. T. Saito and M. A. Lane, eds., Proc. SPIE 5001, 1-15 (2003).

3. E. I. Moses, C. Bibeau, R. E. Bonanno, C. A. Haynam, B. J. MacGowan, R. L. Kauffman, R. W. Patterson Jr., and B. M Van Wonterghem, "The National Ignition Facility: The world's largest laser," in

4. E. I Moses, "The National Ignition Facility: Status of the World's Largest and Most Complex Optical System," in

5. E. I. Moses, "Progress on the NIF," in

6. Campbell, J. H., Hawley-Fedder, R. A., Stolz, C. J., Menapace, J. A., Borden, M. R., Whitman, P. K., Yu, J., Runkel, M., Riley, M. O., Feit, M. D. and Hackel, R. P., "NIF optical materials and fabrication technologies: an overview," in Optical Engineering at LLNL II: The National Ignition Facility, C. R. Wuest and M.A. Lane, eds., Proc. SPIE 5341, 84-101 (2004).

7. J. H. Campbell "Nd-doped Phosphate Glasses for High-Power/High-Energy Lasers," 2004.

8. C. J. Stolz, C. L. Weinzapfel, A. L. Rigatti, J. B. Oliver, J. Taniguchi, R. P. Bevis, and J. S. Rajasansi, "Fabrication of meter-scale laser resistant mirrors for the National Ignition Facility, a fusion laser," in Advances in Mirror Technology for X-ray, EUVL, Lasers and Other Applications, A. M. Khounsary, U. Dinger, and K. Ota, eds., Proc. SPIE 5193, 50-58 (2003).

9. Stolz, C. J., Hafeman, S. and Pistor, T. V., "Light intensification modeling of coating inclusions irradiated at 351 and $1053 \mathrm{~nm}$," in Appl. Opt., submitted. 
10. Stolz, C. J., Feit, M. D. and Pistor, T. V., "Laser intensification by spherical inclusions embedded within multilayer coatings," in Appl. Opt. 45, 1594-1601 (2006).

11. Norton, M. A., Stolz, C. J., Donohue, E. E., Hollingsworth, W. G., Listiyo, K, Pryatel, J. A. and Hackel, R. P., "Impact of contaminates on the laser damage threshold of 1 HR coatings," in Laser-Induced Damage in Optical Materials: 2005, G. J. Exarhos, A. H. Guenther, K. L. Lewis, D. Ristau, M. J. Soileau, and C. J. Stolz, eds., Proc. SPIE 5991, 59910O-1-9 (2005).

12. Stolz, C. J., Adams, J., Shirk, M. D., Norton, M. A., and Weiland, T. L., "Engineering meter-scale laser resistant coatings for the near IR," in Advances in Optical Thin Films II, C. Amra, N. Kaiser, and H. A. Macleod, eds., Proc. SPIE 5963, 59630Y-1-8 (2005).

13. Gourdin, W. H., Dzenitis, E. G., Martin, D. A., Listiyo, K., Sherman, G. A., Kent, W. H., Butlin, RK., Stolz, C. J., and Pryatel, J. A. "In-situ surface debris inspection and removal system for upward-facing transport mirrors of the National Ignition Facility," in Laser-Induced Damage in Optical Materials: 2004, G. J. Exarhos, A. H. Guenther, K. L. Lewis, N. Kaiser, M. J. Soileau, and C. J. Stolz, eds., Proc. SPIE 5647, 107-117 (2005).

14. Stolz, C. J., Genin, F. Y., and Pistor T. V., "Light intensification by nodular defects in multilayer coatings," in Optical Interference Coatings on CD-ROM, (The Optical Society of America, Washington DC,TuF9 (2004)

15. Stolz, C. J., Runkel, M., McBurney, M. S., Cheek, R. E., and Menapace, J. A., "Metrology of mirrors for the National Ignition Facility," in Optical Engineering at LLNL II: The National Ignition Facility, C. R. Wuest and M.A. Lane, eds., Proc. SPIE 5341, 114-120 (2004).

16. Boland, R., "Computer control and monitoring of electrolytic in-process dressing of metal bond fine grinding wheels for NIF optics," Proc. SPIE 3782 (1999). 